\title{
Return on Investment for Open Source Hardware Development
}

\author{
J.M. Pearce ${ }^{a^{*}}$
}

a. Department of Materials Science \& Engineering and Department of Electrical \& Computer

Engineering, Michigan Technological University, MI, 49931, USA

* corresponding author: 601 M\&M Building, 1400 Townsend Drive, Houghton, MI 49931-1295

\section{pearce@mtu.edu}

\begin{abstract}
The availability of free and open source hardware designs that can be replicated with low-cost 3-D printers provide large values to scientists that need highly-customized low-volume production scientific equipment. Digital manufacturing technologies have only recently become widespread and the return on investment (ROI) was not clear, so funding for open hardware development was historically sparse. This paper clarifies a method for determining an ROI for FOSH scientific hardware development. By using open source hardware design that can be manufactured digitally the relatively minor development costs result in enormous ROIs for the scientific community. A case study is presented of an syringe pump released under open-licenses, which results in ROIs for funders ranging from 100 s to 1,000 s of percent after only a few months. It is clear that policies encouraging free and open source scientific hardware development should be made by organizations interested in maximizing return on public investments for science.
\end{abstract}

\section{Introduction}

As prominent voices in the scientific research community have pointed out, funding cuts continue to reduce grant success rates [1,2]. This creates hyper-competitiveness with the concomitant diminishing of risk taking and innovation among researchers of all ages and desertion of many young investigators [1,2]. In addition, researchers consume an ever-increasing fraction of their time with grant writing rather than actually doing science. Simultaneously, the national average overhead charged on grants (indirect costs primarily used to subsidize administrative salaries and building depreciation), has climbed to 52\% [3], which further limits scientists' ability to do research with the hard-earned funding they do obtain. Experimentalists are perhaps the hardest hit as low-volume, highly-specialized equipment needed to push further scientific progress continues to demand premium and often shockingly high prices on the market.

Fortunately, advances in low-cost electronics and 3-D printing [4] enables a new paradigm of scientific equipment production where scientists in the developed and developing worlds can fabricate tools themselves from digital plans [5-9]. There has been an exponential rise in designs for hardware released under open-source, creative commons licenses or placed in the public domain [10] including the 3-D printers themselves [4]. This free and open source hardware (FOSH or open hardware) is shared between scientists by providing the bill of materials, schematics, assembly instructions, and procedures needed to fabricate a digital replica of the original [9]. FOSH reduces redundant problem solving in laboratories around the world, accelerates innovation due to rapid laterallyscaled feedback [5-9]. In this way hardware can benefit from the same methods that have proven so successful with free and open source software [11-12]. The number and variety of FOSH scientific tools is rapidly expanding $[6,9,13-20]$. For scientists that need access to highlycustomized low-volume tools the open source method and digital replication can result in significant cost savings $[6,9,13]$. Although research capital goes farther with digitally distributed open designs, it still costs money to develop them. This paper investigates the return on investment (ROI) possible for fund agencies such as the NIH or NSF for developing open hardware for science. A brief case study is provided to illustrate the economic calculations and then policy recommendations are made to maximize the ROI in research and development in any field accessible to open hardware design. 


\section{Calculations}

The value obtained from a FOSH design can be determined from the downloaded substitution valuation [21] $\left(\mathrm{V}_{\mathrm{D}}\right)$ at a given time based on the number of downloads $\left(\mathrm{N}_{\mathrm{D}}\right)$ for a given design at time, $\mathrm{t}$ :

$$
V_{D}(t)=\left(C_{p}-C_{f}\right) \times P \times N_{D}(t)
$$

Where $\mathrm{C}_{\mathrm{p}}$ is the cost to purchase a traditionally manufactured product, $\mathrm{C}_{\mathrm{f}}$ is the marginal cost to fabricate it digitally (e.g. open-source 3-D printing), and $\mathrm{P}$ is the percent of downloads resulting in a product. It should be pointed out that $\mathrm{P}$ is subject to error as downloading a design does not guarantee manufacturing. On the other hand, a single download could be fabricated many times, traded via email, memory stick or posted on $\mathrm{P} 2 \mathrm{P}$ websites that are beyond conventional tracking. The savings are maximized for custom low-volume scientific equipment where $C_{f}$ is generally only $1-10 \%$ of $C_{p}[9,17]$, creating a 90-99\% savings.

Investment in scientific open source hardware can thus create a return on investment that can be calculated by:

$$
R O I=\frac{V_{D}(t)-I}{I}
$$

where I is the cost of the investment in the development of the FOSH scientific tool.

\section{Case Study}

Consider the case study of a simple open-source syringe pump library design [22], which may be government funded for scientific innovation acceleration, but also have applications in STEM education and medicine. The lowcost open source pumps are completely customizable allowing both the volume and the motor to scale for specific applications such as any research activity including carefully controlled dosing of reagents, pharmaceuticals, and other applications. The design, bill of materials and assembly instructions are globally available to anyone wishing to use them.

The pump library was designed using open-source and freely available OpenSCAD, which is script-based, parametric CAD package enabling scientists to customize the design for themselves [22]. The majority of the pump parts can be fabricated with an open-source RepRap 3-D printer and readily available parts such as a stepper motor and steel rods. The pumps can be used as wireless control devices attached to an open-source Raspberry Pi computer.
Performance of the syringe pumps generated by the open source library were found to be consistent with the quality of commercial syringe pumps [22].

As of this writing (Feb. 2015) the designs for the open source pump, which were released in Sept. 2014, have been downloaded from two digital repositories a total of $\mathrm{N}_{\mathrm{D}}=$ 1035 times (224 on Thingiverse [23] and 811 on Youmagine [24]). The cost to purchase a traditionally manufactured syringe pump, $\mathrm{C}_{\mathrm{p}}$, ranges from $\$ 260-\$ 1,509$ for a single pump and $\$ 1,800-\$ 2606$ for a dual pump [22]. $\mathrm{C}_{\mathrm{f}}$ for the materials for an open source syringe pump is $\$ 97$ and $\$ 154$ for the single and double pump, respectively [22]. The time to assemble either the single or double pump is less than an hour and can be accomplished by a non-expert. Although the time to print the components is less than four hours on a conventional RepRap, workers can do other tasks while printing. The assembler hourly rate is assumed to be $\$ 10 /$ hour because no special skills are needed. $\mathrm{P}$ was assumed to be 1 as informal discussions with 50 RepRap owners found that the vast majority of designs downloaded were printed. This is because although RepRap owners may view many designs they only download the STL files (STereoLithography is a file format used for 3-D printers) of the designs they intend to print. This provides a savings for substituting the open source pump for a commercial one of $\$ 153$ to $\$ 1,402$ for a single and $\$ 1,636$ to $\$ 2,442$ for the double pump. Thus, following equation 1 the value $V_{D T}$ of the pump library on Feb. 5, 2014 ranged from over $\$ 168,000$ to over $\$ 2.5$ million.

The investment needed to create the pump library was trivial in comparison to the savings. The mechanical designs were completed by experienced engineers in $<6$ worker-hours. To print and revise the five 3-D printed components took 3 hours, assembly less than 1 hour and software development and Pi wiring less than 16 hours. The total design and prototyping time was less than 26 hours total. This schedule was possible because the designers were experienced with similar designs and had access to all of the components. Let us assume that the pump design was completed as part of an NSF or NIH grant that involved overhead, materials, validation testing from a $\mathrm{PhD}$ student over a semester thus involving an initial investment, I, of $\$ 30,000$. By equation 2 this provides ROIs ranging from $460 \%$ to over $8,300 \%$.

These ROIs, however, are conservative as the value of the FOSH in labs is not only the amount saved for research directly, but also includes the value of the overhead (indirect costs) charged on grants to purchase the product 
commercially. When the national average overhead rate of $52 \%$ is included on the cost of conventional equipment the ROIs increase to range from over $750 \%$ to over $12,000 \%$, respectively.

\section{Discussion}

Some care must be taken in evaluating these ROIs. First, to be conservative it should be pointed out that although the majority of down loaders can be presumed to be American, not all of the savings would accrue to those that U.S. government funding agencies are supporting. Although, improving science globally will help researchers in the U.S. indirectly it may not be viewed as a direct ROI. On the other hand, the low end value is from a simple infusion pump with considerably less functionality than the FOSH syringe pumps. It is a safe assumption that the majority of down loaders are likely to be replacing more sophisticated scientific devices. In addition, it also is clear that the number of downloads and fabrications of the syringe pumps will continue to expand with time providing ever increasing value for the scientific community and higher ROIs for funding such development.

There are other benefits as well. As the pump meets the standards for research and has already been vetted it seems reasonable that it would be most likely to be adopted university labs first. This would results in potential additional value for both scientific research and education. By decreasing the costs of research equipment more of the diminishing resources for science are available to do science. For example, if a quad syringe pump is fabricated for a molecular biology lab the savings would be enough to hire a summer undergraduate researcher - thus presumably increasing the scientific discovery rate. In addition, as the designs are $\mathrm{FOSH}$, other labs are already improving the tool and re-sharing the improvements (e.g. [25]). This creates superior scientific equipment in the future with no additional expenditures. Similarly, due to lower costs, FOSH could be used in classroom or lab courses at every level, thus improving education.

Quantifying the value of an increased rate of discovery in science and medicine because of lower costs or superior equipment or better education entails specific detailed studies. These studies, for example, could utilize follow-up surveys targeted at all down loaders of the design and STL files to get a precise account of $P, C_{p}$ and $C_{f}$. Then, in turn these initial calculated ROIs could be augmented by following the results of groups/schools that adopted the open hardware. This would be useful for obtaining exact values of ROI. However, the methodology used here provides reasonably accurate values based on conservative assumptions of simply replacing proprietary equipment. Qualitatively, it is clear that free and open source scientific hardware has ROIs set by the minimum calculated by equations 1 and 2 and these are significantly higher than those involving proprietary investment by public funding groups.

It should also be noted that as free and open source hardware becomes more commonplace in the scientific establishment there may be a cultural shift within labs to employ 'makers' to build and troubleshoot open source equipment. The 'maker movement' is a growing culture of hands-on making, creating, designing, and innovating at all ages [26,27]. Such troubleshooting is already provided in a limited way by technicians, research assistant students and research scientists, but would be expected to expand and provide positions for the growing number of makers as more scientific hardware becomes completely accessible and able to be customized.

Finally, it should be pointed out that there is nothing at all remarkable about the syringe pump used as an example here. Any one of dozens of other existing open source scientific hardware designs that have a $\mathrm{C}_{\mathrm{f}}$ that is a tiny fraction of $C_{p}[9,17]$ and that have been downloaded a small number of times would show similar enormous ROIs. In addition, there are numerous types of equipment that could be redesigned as FOSH with low fabrication costs that could be widely applied outside of scientific labs (e.g. hand held nitrate testers for water quality or use for farmers to determine fertilizer requirements) that would have enormous potential returns for society.

For entities such as the NIH, which are trying to leverage their investments for the greatest common good, FOSH development has extremely high potential ROIs. Traditional investments in proprietary development would not come close to such a return, nor would the source be available for others to immediately begin working on because of the 20-year external innovation hiatus demanded by the current patent system [28].

It is clear that federal funding (such as through the NIH, NSF, DOE, DOA, DOD, and NASA, etc.) should be prioritized for the development of open-source scientific hardware because of the enormous potential ROIs for the nation's scientific community. This can be accomplished with a combination of traditional CFPs for academic grants and programs like SBIR and STTR programs. In addition, 
the U.S. can run national contests like the X-prize or "first to make" specific technical goal "bounties".

Just as proprietary tools on the market are, all FOSH scientific designs should be vetted, tested and validated. This will largely eliminate the technical risks for labs to adopt the use of the hardware, while at the same time ensuring that scientific equipment no longer becomes obsolete as proprietary systems can when a company loses key personnel, discontinues a product line or goes out of business. Funding for this validation would be the majority of the cost of scientific FOSH. Then, in order to ensure the widest distribution of the free technologies, NIH's 3D Print Exchange should be expanded to act as a national free online catalog of tested, vetted and validated free and opensource scientific hardware. The NIH's 3D Print Exchange should be augmented to be able to house the bill of materials, digital designs, instructions for assembly and operation, and the source code for all software and firmware.

\section{Conclusions}

By funding open source hardware design that can be manufactured digitally, the relatively minor development costs results in enormous ROIs for the scientific community. As the designs are reusable, with solid modeling and 3-D printing, designs can be expanded or joined together rapidly increasing the rate of innovation. The case study presented here found ROIs of 100 s to 1000 s of percent from a relatively simple scientific device being released under open-licenses. It is clear that free and open source scientific hardware development should be funded by organizations interested in maximizing return on public investments.

\section{Funding}

This work was supported by the National Science Foundation [NSF STTR Phase I Grant \#IIP-1417061].

\section{Conflict of Interest}

None

\section{References}

[1] 'No money, no research'. (2014) Nature Methods 11: 1077-1077.

[2] Alberts, B., Kirschner, M.W., Tilghman, S., Varmus, H. (2014) 'Rescuing US biomedical research from its systemic flaws'. Proceedings of the National Academy of Science USA 111: 5773-5777.
[3] Jan T. (2013) 'Research giants win on federal funding'. Boston Globe. March 18, 2013.

[4] Jones, R., Haufe, P., Sells, E. (2011) 'RepRap - the replicating prototyper'. Robotica. 29/1:177-191.

[5] Jones, N. (2012) 'Science in three dimensions: the print revolution'. Nature 487: 22-23.

[6] Pearce, J.M. (2012) 'Building Research Equipment with Free, Open-Source Hardware'. Science 337/6100: 13031304.

[7] 'Sharing blueprints for better research'. (2013) Nature Methods 10: 591-591.

[8] Pearce, J.M., Blair, C., Laciak, K., Andrews, R., Nosrat, A., Zelenika-Zovko, I. (2010) '3-D printing of open source appropriate technologies for self-directed sustainable development'. Journal of Sustainable Development 3/4:1729.

[9] Pearce, J.M. (2014) 'Open-Source Lab: How to Build Your Own Hardware and Reduce Research Costs'. Elsevier: New York.

[10] Wittbrodt, B., Glover, A., Laureto, J., Anzalone, G., Opplinger, D., Irwin, J., Pearce, J. (2013) 'Life-cycle economic analysis of distributed manufacturing with opensource 3-D printers'. Mechatronics 23/6:713-726.

[11] Ball, P. (2003) 'Openness makes software better sooner'. Nature, 030623-6.

[12] Lakhani, K.R., Hippel, E.V. (2003) 'How open source software works: free user-to-user assistance'. Research Policy 32:923- 943.

[13] Pitrone, P. G., Schindelin, J., Stuyvenberg, L., Preibisch, S., Weber, M., Eliceiri, K., Huisken, J., Tomancak, P. (2013) 'OpenSPIM: an open-access lightsheet microscopy platform'. Nature Methods 10: 598-599.

[14] Gualda, E. J., Vale, T., Almada, P., Feijó, J., Martins, G., Moreno, N. (2013) 'OpenSpinMicroscopy: an opensource integrated microscopy platform'. Nature Methods 10: 599-600.

[15] Carvalho, M. C., Eyre, B. D. (2013) 'A low cost, easy to build, portable and universal autosampler for liquids'. Methods in Oceanography 8:23-32.

[16] Wolf, L.K. (2013) 'Print-A-Lab'. Chem \& Eng News 91: 44-45.

[17] Zhang, C., Pearce, J., Faria, R., Anzalone, N. (2013) 'Open-Source 3D-Printable Optics Equipment'. PLoS ONE 8/3, e59840.

[18] Herrmann, K. H., Gartner, C., Gullmar, D., Kramer, M., Reichenbach, J.R. (2014) '3D printing of MRI compatible components: why every MRI research group should have a low-budget 3D printer'. Med Eng \& Physics 36/10:

1373-1380.

[19] Gross, B. C., Erkal, J., Lockwood, S., Chen, C., Spence, D. (2014) 'Evaluation of 3D Printing and Its 
Potential Impact on Biotechnology and the Chemical Sciences'. Analytical Chem 86/7: 3240-3253.

[20] Chiu, S. H., Urban, P. L. (2015) 'Compact 3D-printed interface for coupling open digital microchips with Venturi easy ambient sonic-spray ionization mass spectrometry'. Biosensors and Bioelectronics. 64: 260-268.

[21] Pearce, J.M. (2015) 'Quantifying the Value of Open Source Hardware Development'. Modern Economy 6:1-11. [22] Wijnen, B., Hunt, E., Anzalone, G., Pearce, J. (2014) 'Open-Source Syringe Pump Library'. PLoS ONE 9/9, e107216.

[23] Pearce, J. 'Open Source Syringe Pump'. (2014) Available at: http://www.thingiverse.com/thing:475841 (Accessed: 5th February 2014)

[24] Pearce, J. 'Syringe Pump' (2014) Available at: https://www.youmagine.com/designs/syringe-pump

(Accessed: 5th February 2014).
[25] Lynch, D. Modified Open Source Syringe Pump Parts (2014). Available at:

http://www.thingiverse.com/thing:578010 (Accessed: 5th February 2014)

[26] Dougherty, D. (2012). 'The maker movement.' Innovations, 7(3), 11-14.

[27] Peppler, K., Bender, S. (2013) 'Maker movement spreads innovation one project at a time'. Phi Delta Kappan, 95(3): 22-27.

[28] Pearce, J. M. (2012) 'Make nanotechnology research open-source'. Nature 491: 519-521. 